\title{
Pathology of the Penis in Long-Term Diabetic Rats
}

\author{
K. Fani ${ }^{1}$, A.P.Lundin ${ }^{2}$, M.M. Beyer ${ }^{2}$, F. A.Jimenez ${ }^{1}$ and E. A. Friedman ${ }^{2}$ \\ ${ }^{1}$ Department of Pathology, Brooklyn Veterans Administration Hospital and \\ ${ }^{2}$ Department of Medicine, Downstate Medical Center, State University of New York, Brooklyn, New York, USA
}

\begin{abstract}
Summary. Pathological changes in the penis of long-term diabetic rats ( $>1$ year) include epidermal atrophy and lipid droplets in erectile tissue and dermis, as well as thickening of capillary basement membranes, dilatation and microaneurysms of capillaries, and atrophy and degeneration of erectile smooth muscle. These changes are similar to those previously
\end{abstract}

described as occurring in other organs, but damage to nerves and smooth muscle can best be appreciated with electron microscopy.

Key words: Penis, diabetes mellitus, rat, diabetic complications, diabetic angiopathy
Rat models of chemically induced diabetes have proved useful for studying pathological changes associated with hyperglycaemia. Many retinal and renal changes found in diabetic patients are also noted in rats rendered experimentally diabetic $[1,2]$. We report pathological changes observed in the penis of rats made diabetic by streptozotocin.

\section{Materials and Methods}

Twelve male Lewis rats (Harlan, Indianapolis, Indiana, USA) weighing between $125-160 \mathrm{~g}$ were made diabetic with IV streptozotocin at a dose of $65 \mathrm{mg} / \mathrm{kg}$. All diabetic rats and six age-matched controls were allowed free access to tap water and standard rat chow. No attempt was made to treat the resultant hyperglycaemia by restriction of diet or administration of insulin.

Venous glucose levels were measured with a glucose analyzer (Beckman Instruments, Somerset, New Jersey, USA) on tail blood obtained from normal and diabetic animals in the non-fasting state. Rats with a blood glucose $>22 \mathrm{mmol} / 1$ at $24 \mathrm{~h}$ post-streptozotocin which remained above $16.6 \mathrm{mmol} / 1$ for at least 2 weeks were entered into the study. The diabetic rats only remained in the study if their blood. glucose levels remained at $16.6 \mathrm{mmol} / \mathrm{l}$ or above.

Seven diabetic and six control rats were sacrificed 15 months after streptozotocin treatment. They were killed instantly by decapitation, and all tissues were removed and fixed in 3\% gluteraldehyde. Penises from both control and diabetic rats were processed for light microscopy by standard histological methods and stained with haematoxylin-eosin. Portions of the penis for electron microscopy were postfixed in osmium tetraoxide, dehydrated in graded alcohol and embedded in epon. These sections were cut with a microtome (LKB ultratome III, LKB Instruments, Bromma, Sweden) at $600^{\circ} \mathrm{A}$ and stained with uranyl nitrate and lead citrate.

\section{Results}

Paraffin sections of the penis stained with haematoxylin-eosin and studied under low power $(\times 10)$ did not reveal any notable differences between normal and diabetic rats at 15 months. Under higher power $(\times 180)$, the stratified squamous epithelium was markedly attenuated in diabetic rats (3-4 cells) compared with that of non-diabetics rats ( $7-13$ cells, Figs. 1 and 2 ). Wide spacing of cellular elements and collagen bundles, changes indicative of oedema were apparent in the upper dermis of diabetic rats (Fig.3). Aneurysmal dilation of capillaries, a common pathological finding in other tissues of diabetic rats, was present in the penis of rats with longstanding diabetes (Fig. 4).

Morphological changes were also found in erectile tissue (Figs. 5 and 6). In the non-diabetic rats, the sinuses were thickly lined with layers of smooth muscle while in the diabetic rats, the muscle layers are sparse. A striking finding in the diabetic rats was the presence of lipid droplets throughout the upper dermis and corpus cavernosum (Figs. 3 and 6).

Under light microscopy, no morphological changes were seen in myelinated nerves. However, under electron microscopy degenerative axonal changes are noted (Figs. 7 and 8). Similar destruction of fibres were noted in unmyelinated nerves (Figs. 9 and 10).

Capillary basement membranes of the diabetic animals were thickened, lamellated and smudged (Fig.11). 

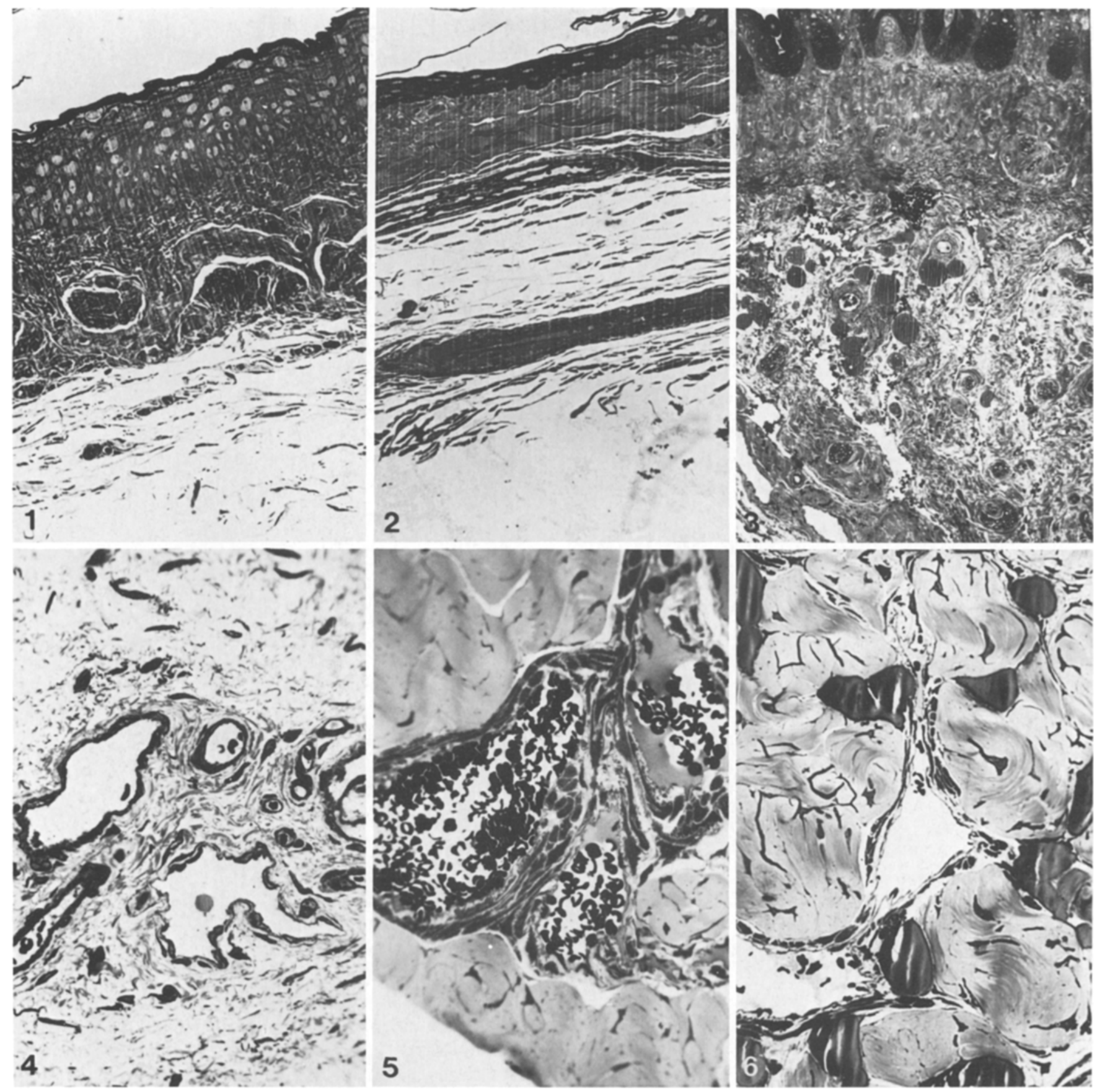

Fig. 1. Penile skin of non-diabetic rat. The keratinized stratified squamous epithelium has several layers of keratinocytes $(\times 139)$

Fig. 2. Penile skin of diabetic rat. The epidermis consists of a few layers of cells $(\times 139)$

Fig. 3. Erectile tissue of diabetic rat showing oedema with many lipid droplets scattered in oedematous tissue $(\times 62)$

Fig.4. Diabetic rat penis showing aneurysmal dilation of capillaries and proliferation of the capillary endothelium $(\times 328)$

Fig.5. Erectile tissue of normal rat. Layers of plump smooth muscle line the sinuses $(\times 328)$

Fig. 6. Erectile tissue of diabetic rat. Smooth muscle cells lining the sinuses are few in number and thin. Lipid droplets are seen within the connective tissue $(\times 328)$ 


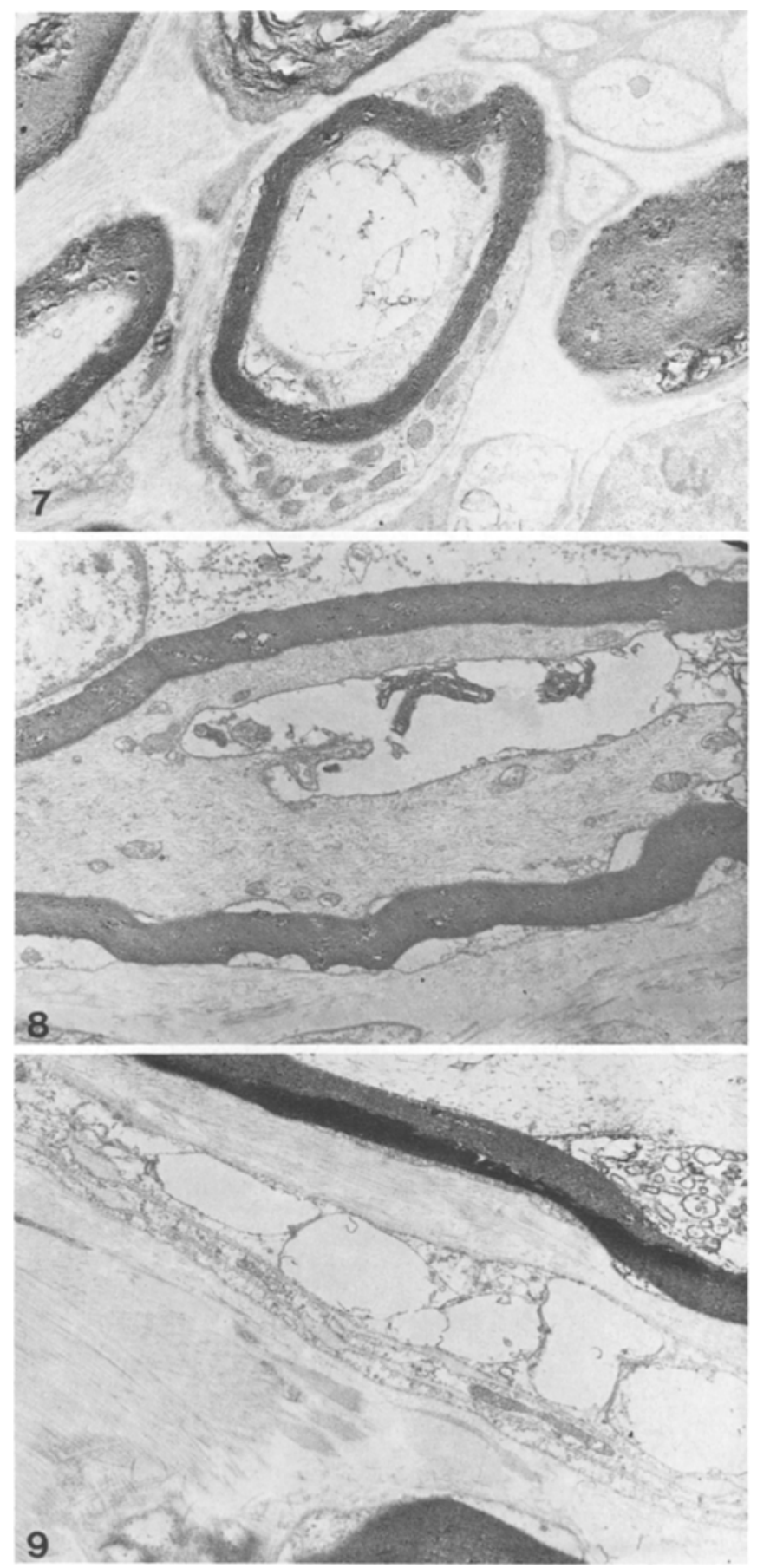

Fig. 7. Transverse section of myelinated nerve in penis of diabetic rat. Axonal degeneration is demonstrated $(\times 8290)$

Fig. 8. Longitudinal section of myelinated nerve, showing degeneration of axonal filaments, tubules and mitochondria $(\times 8290)$

Fig. 9. Longitudinal section of unmyelinated nerve showing destruction of almost all axonal structures $(\times 8290)$

The cytoplasm of smooth muscle cells in the erectile tissue of 15-month-old normal rats was almost entirely filled with myofibrils (Fig. 12), while thinning, fragmentation and destruction of myofilaments were noted in chronically diabetic rats (Fig. 13).

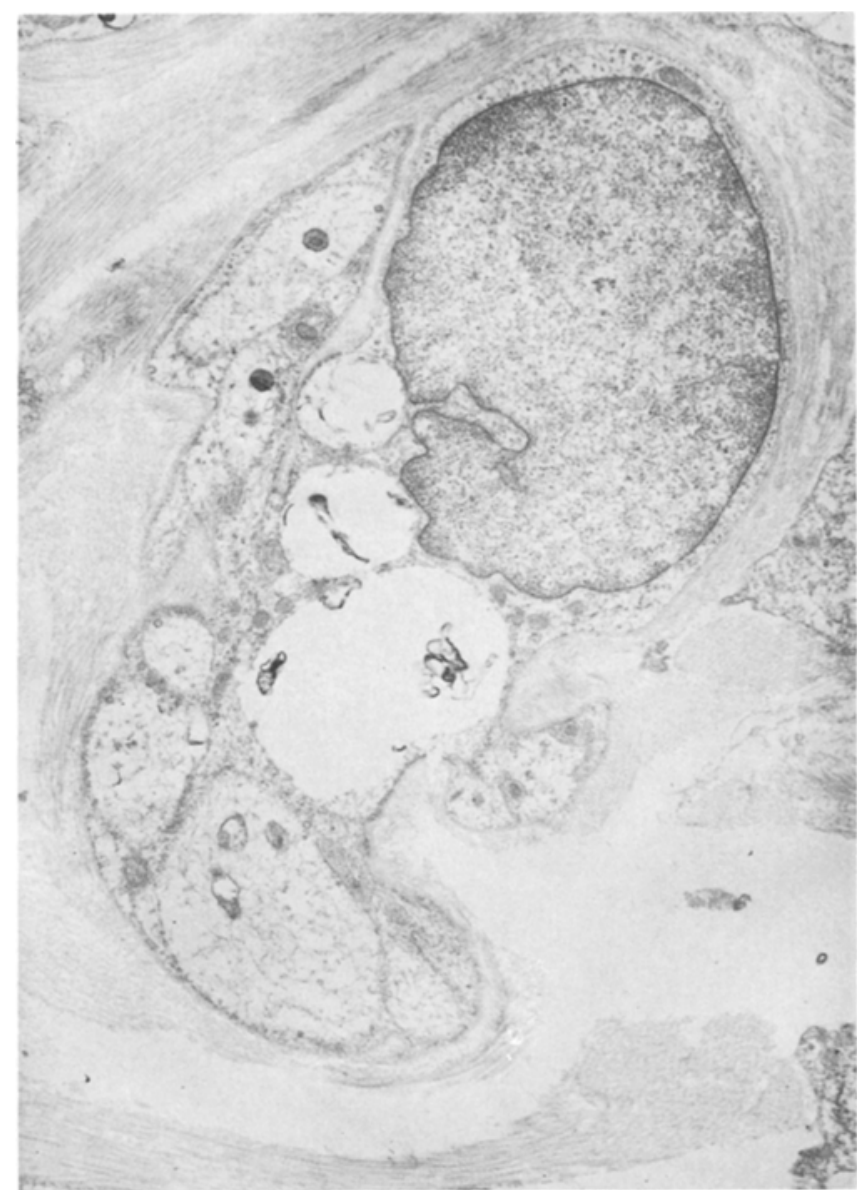

Fig. 10. Transverse section of unmyelinated nerve where a hollow shaft represents the remains of three axons $(\times 8290)$

\section{Discussion}

Advances in understanding the pathological effects of diabetes mellitus on retinae and kidneys have been achieved through the use of rat models of induced diabetes [2]. Other organs in diabetic rats have not been evaluated as thoroughly as models for the study of diabetic complications. Small vessel damage is a hallmark of diabetes affecting almost all the tissues of the body which may result in loss of vision and impaired renal function. Sexual impotence in male diabetic patients may cause at least as much concern psychologically. Impotence may affect up to $50 \%$ of diabetic men [3-6]. The cause of sexual dysfunction in diabetic patients is still in some dispute, although erectile dysfunction may be due to penile nerve or blood vessel damage. We examined the penis in streptozotocin-induced diabetic rats to determine the extent of neurological and vascular damage associated with long-standing hyperglycaemia.

By light and electron microscopy, we observed that penises of diabetic rats undergo severe pathological changes after one year. We identified among these changes epidermal atrophy, lipid droplets in erectile tissue and dermis, thickening of capillary basement mem- 

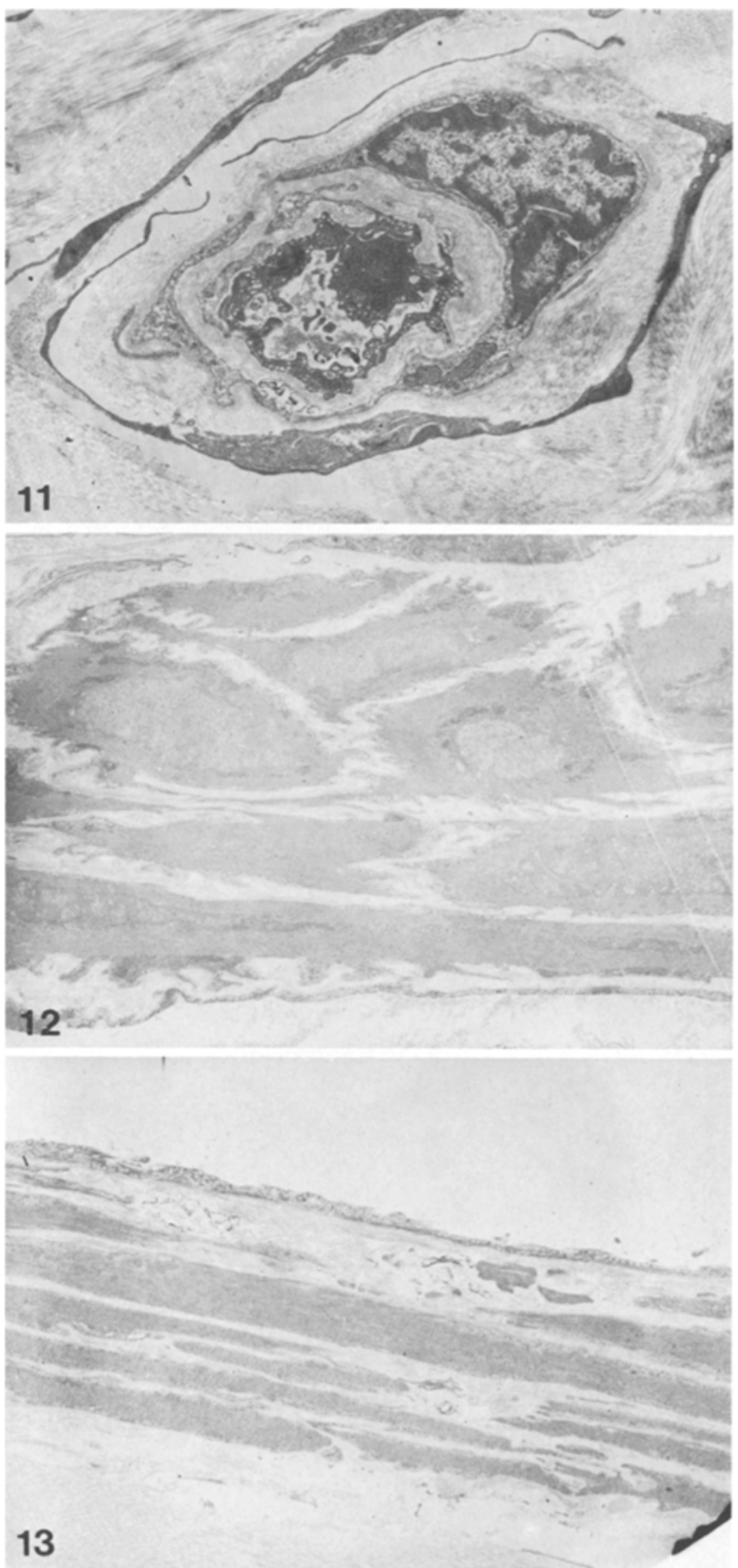

Fig. 11. Penile capillary of diabetic rat with marked thickening, lamellation and smudging of the basement membrane $(\times 4675)$

Fig. 12. Erectile smooth muscle of control rat. Myofibrils fill entire cytoplasm $(\times 4675)$

Fig. 13. Erectile smooth muscle in diabetic rat. Fragmentation and thinning of muscle fibres $(\times 4675)$

branes, dilatation and microaneurysms of capillaries, and atrophy and degeneration of erectile smooth muscle. Destruction of myelinated and unmyelinated nerves was detectable only by electron microscopy. Many of these changes have been described in other organs in both diabetic men and rats.

We do not know if our diabetic or non-diabetic rats had any sexual dysfunction, but it is known that diabetic men with advanced neurological and vascular disease similar to that seen in our rats have a high incidence of impotence. Ellenberg [7] reported that 37 out of $45 \mathrm{im}-$ potent diabetic men had neurogenic bladder abnormalities, while only three of 30 potent diabetic subjects had bladder dysfunction. Because the same autonomic nerves control both erectile function of the penis and micturition, it is likely that both functions may be affected simultaneously. Faerman et al. [8] examined penile biopsies from impotent diabetic patients and, under light microscopy, identified damage to autonomic nerve fibres. Morphological alterations seen with silver stains, but not with haematoxylin and eosin, include diffuse thickening of nerve fibres, some of which exhibited beading, vacuolization, and rigidity, with abnormalities of fibre caliber. None of these findings was seen in biopsies from control age-matched potent normal subjects. We concluded that diabetic impotence is likely to be due to a lesion of the nerve fibres that control erection. Melman et al. [9], on the other hand, failed to detect pathological changes by light microscopy in either myelinated or unmyelinated nerve fibres of the penis in impotent male diabetic patients. We have shown that damage to these nerves may only be seen at higher magnifications.

Vascular damage may also correlate with diabetic impotence due to restricted blood flow into the cavernous sinuses [10]. In non-diabetic patients, erection can be impaired as a result of atherosclerotic vascular changes [11]. The atrophy and degeneration of erectile smooth muscle cells that we noted with electron microscopy in diabetic rats could contribute to impotence if similarly present in diabetic men. McCulloch et al. [6] found that patients with severe microangiopathy and autonomic neuropathy were likely to be impotent.

Recent evidence suggests a role for vasoactive intestinal peptide (VIP) in the nervous control of male external genitalia [12]. High concentrations of VIP were found by immunocytochemistry exclusively in fine autonomic nerves around the pudendal arteries and erectile tissues of the corpus cavernosum. Diminution of VIP levels in the penile tissues of impotent diabetic subjects or animals would be suggestive evidence of a neurological basis for impotence.

Whether or not male rats with long-standing diabetes are impotent is to be determined. Nevertheless, because of the similar morphological alterations seen in the penises of both impotent diabetic men and longterm streptozotocin-diabetic rats, in addition to the further damage we have seen with electron microscopy, we suggest that this model may be suitable for examining the pathology of diabetic impotence.

Acknowledgements. We wish to acknowledge the invaluable assistance of E.Schneider in the preparation of all pathological materials. 


\section{References}

1. Heath $H(1970)$ Experimentally induced retinopathies and diabetes. Br Med Bull 26: 151-155.

2. Mauer SM, Steffes MW, Brown DM (1979) Animal models of diabetic nephropathy. Adv Nephrol 8: 23-42

3. Rubin A, Babbott D (1958) Impotence and diabetes mellitus. JAMA 168: 498-500

4. Schoffling K, Federling K, Ditschunheit H, Pfeiffer, EF (1963) Disorders of sexual function in male diabetics. Diabetes 12: 519-527

5. Kolodny RC, Kahn CB, Goldstein HH, Barnett DM (1974) Sexual dysfunction in diabetic men. Diabetes 23: 306-309

6. McCulloch DK, Campbell IW, Wu FC, Prescott RJ, Clarke BF (1980) The prevalence of diabetic impotence. Diabetologia 18: 279-283

7. Ellenberg $M$ (1971) Impotence in diabetes: The neurologic factor Ann Intern Med 75: 213-219

8. Faerman I, Glocer L, Fox D, Jadzinsky MN, Rapaport M (1974) Impotence and diabetes: Histological studies of the autonomic nervous fibers of the corpora cavernosa in impotent diabetic males. Diabetes 23: 971-976

9. Melman A, Henry DP, Felten DL, O'Connor B (1980) Effect of diabetes upon penile sympathetic nerves in impotent patients. South Med J 307-309

10. Cohen MS, Sharpe W, Warner RS, Zorgniotti A (1980) Morphology of corporal cavernosa arterial bed in impotence. Urology 16: 382-385

11. Ruzbarsky V, Michal V (1977) Morphologic changes in the arterial bed of the penis with aging. Invest Urol 15: 194

12. Polak JM, Gu J, Mina S, Bloom SR (1981) Vipergic nerves in the penis. Lancet ii: 217-219

Received: 31 August 1982

and in revised form: 17 June 1983

Dr. A. Peter Lundin

Downstate Medical Center

450 Clarkson Avenue, Box 52

Brooklyn, New York 11203, USA 